\title{
The Impact of a Pre-Dialysis Educational Program on the Mode of Renal Replacement Therapy in a Saudi Hospital: A Retrospective Cohort Study
}

Ahlam A. Alghamdi ${ }^{1}$, Khalid A. Almotairy ${ }^{2}$, Roqaya Moeedh Aljoaid ${ }^{1}$, Nedaa Anwar Al Turkistani ${ }^{1}$, Rawan Walid Domyati ${ }^{1}$, Morsy Mohamed Morsy Abdelrahman ${ }^{3}$, Kholod Samer Shobain ${ }^{1}$, Cathariena M. Uys ${ }^{4}$

1. Health Education Department, King Fahad Armed Forces Hospital, Jeddah, SAU 2. Family Medicine: Health Education Department, King Fahad Armed Forces Hospital, Jeddah, SAU 3. Nephrology Department, King Fahad Armed Forces Hospital, Jeddah, SAU 4. Nursing: Quality Department, King Fahad Armed Forces Hospital, Jeddah, SAU

Corresponding author: Ahlam A. Alghamdi, ahlam_gh89@hotmail.com

\section{Abstract \\ Background}

Self-care and peritoneal dialysis (PD) benefits have been underutilized in patients with end-stage renal disease (ESRD). The pre-dialysis education program (PDEP) has been generally introduced as an acceptable tool in increasing the rates of PD and has been reportedly recommended for ESRD patients as part of the introduced care. We aim to study the effect of PDEP on ESRD and whether they would prefer PD of centerbased hemodialysis (HD).

\section{Methods}

This is a retrospective cohort study that was done at King Fahad Armed Forces Hospital in Jeddah, Saudi Arabia, in the dialysis center. Data were collected on patients and included demographics, preference of renal replacement therapy modality, and other possible factors that may affect patient choices such as educational level, economic status, and age.

\section{Results}

A total of 213 ESRD patients that met our criteria were included, with a total of 75 patients receiving PDEP. Out of those who received the PDEP, $57.3 \%$ and $42.7 \%$ of patients decided to perform HD and PD, respectively. There was a significant impact of PDEP on reducing HD choice [OR $(95 \% \mathrm{CI})=0.11(0.05-0.24)$; P-value $<0.001$ ]. Infections did not occur in $50.5 \%$ of the included patients while $45.8 \%, 3.3 \%$, and $0.5 \%$ had central line-associated bloodstream infection (CLABSI), other infections, and peritonitis, respectively. Most of the PD patients (81.8\%) did not have an infection as compared to $42.3 \%$ of the HD patients. HD was also associated with increased admission days [OR $(95 \% \mathrm{CI})=1.27(1.07-1.51)$; P-value $=0.007]$.

Review began 11/15/2020 Review ended 12/02/2020 Published 12/08/2020

\section{() Copyright 2020}

Alghamdi et al. This is an open access article distributed under the terms of the Creative Commons Attribution License CC-BY 4.0., which permits unrestricted use, distribution, and reproduction in any medium, provided the original author and source are credited.

\section{Conclusion}

We found that PDEP positively impacted the rate of PD while PD was associated with favorable outcomes and lower infection rates, emphasizing the importance of an educational program.

Categories: Medical Education, Nephrology, Epidemiology/Public Health

Keywords: peritoneal dialysis, hemodialysis, end-stage renal disease, pre-dialysis education

\section{Introduction}

Incidence rates are growing and have reached up to 6\%-8\% in end-stage renal disease (ESRD) globally [1]. In the Kingdom of Saudi Arabia (KSA), the rate has been also reportedly increasing, as the Saudi Center for Organ Transplantation (SCOT) previously estimated that 19,659 patients underwent either hemodialysis (HD) or peritoneal dialysis (PD), with the largest proportion favoring HD (18,270 patients) in 2017 [2-3]. Favoring either of the renal replacement therapy (RRT) modalities is hugely variable among the different healthcare systems managing ESRD patients worldwide. Many factors have been involved in this preference and are usually non-medical. For example, the availability of resources and trained personnel, bias from physicians, financial aspects, and patients' cultures and habits may influence the choice of one modality over the other [4].

When comparing hospital-based HD to self-dialysis, which includes PD and home-based HD, self-care dialysis has been associated with better outcomes regarding patients' quality of life (QoL) in addition to huge reductions concerning financial burdens [5-7]. Previous reports usually referred to PD as the only selfcare dialysis modality, with reported favorable outcomes [8-11]. Although many investigations have previously reported that the potential use and benefit from PD are generally underestimated, many benefits 
have been reported regarding its early use in an integrated way with other modalities [12-14]. The low rate for using PD is probably due to structural obstacles, nephrologists' attitudes, together with the reduced awareness of chronic kidney disease (CKD) patients towards this modality [15-16]. Therefore, boosting patients' awareness of this mode can lead to an increased prevalence of patients undergoing PD.

The pre-dialysis education program (PDEP) has been generally introduced as an acceptable tool in this field and has been reportedly recommended for ESRD patients as part of the introduced care. The application of this program leads to delivering informative data to the patients and increases their awareness of the most suitable modality to follow, recommendations about lifestyle modifications, and the best treatment regimen to follow. Moreover, many investigations have reported that applying PDEP was generally associated with lower mortality rates by reducing the need to conduct urgent dialysis because of a higher awareness status of patients [17]. Although many studies have demonstrated the efficacy of PDEP on increasing patients' awareness [9-10], many factors related to patients' preference and selection of the best modality based on their QoL are still vague. In this study, we aim to study the impact of conducting PDEP on patients' choice regarding the preference of using PD over HD and the determinants of such preference.

\section{Materials And Methods}

\section{Study design}

This is a retrospective cohort study that was conducted using the data of all patients who started renal replacement therapy either by hemodialysis or peritoneal dialysis and were part of the renal education program in King Fahad Armed Forces Hospital in Jeddah, Saudi Arabia. The data included all patients one year before and one year after the start of the PDEP in March 2019. The educational program was constructed according to the Bagnis et al. program that was published in 2015 [18].

\section{Study population}

The rate at which patients chose peritoneal dialysis in ESRD patients who received PDEP was compared to those who did not receive PDEP over a period of two years (one year before and one year after the start of PDEP. All patients with complete files were included in the study. Meanwhile, patients who started renal replacement therapy outside our hospital or those with an emergency, unplanned initiation of hemodialysis were excluded. A comparison of baseline characteristics between the patient cohorts prior to the introduction of the education program and the group after implementation was illustrated to assess matching.

\section{Data collection tool}

Data were collected utilizing a pre-designed data collection form. The collected data included patient demographic data, choice of renal replacement therapy modality, educational level, economical status, and age. The possible confounding bias was dealt with using an appropriate statistical analysis through multivariate analysis. The source of the data was the patients' medical files which were available for all health educators. Any missing data were acquired through a telephone call or a direct interview with the patient when possible.

\section{Statistical analysis}

All data were analyzed using R software version 4.0.2 [19] using the packages (Rcmdr) [20] and (glm2) [21]. We represent nominal variables as frequencies and percentages by conducting the chi-square test (or Fisher's exact test), as appropriate, to test the difference, according to the presence or absence of PDEP. Furthermore, we used multivariate logistic regression to identify any possible association between PDEP and choice of dialysis modality [22]. In addition, we measured the impact of dialysis modality on patient outcomes [22]. In the multivariate model, the data were adjusted for patients' age, gender, socioeconomic status, education, and the personnel providing the program. Regression results were expressed as odds ratios (ORs) and $95 \%$ confidence interval $(95 \% \mathrm{CI})$. For all statistical tests, P-value $<0.05$ was considered statistically significant.

\section{Results}

\section{Baseline sociodemographic characteristics}

A total of 213 patients were included in the current study; out of them, 35.2\% were provided with PDEP and the other $64.8 \%$ were not. Nearly half of the patients (46.9\%) were greater than 61 years old and $43.7 \%$ of them were males while most of them were either coming from a low-class $(45.1 \%)$ or middle-class $(48.8 \%)$ socioeconomic status. The majority of the patients (94.3\%) earned 5,000 or more Saudi Riyals per month and most of them were residing in Jeddah City (84.0\%). Regarding the educational level among the included patients, $12.2 \%$ had a college degree or higher, $46.9 \%$ had a high school degree or less, and $40.8 \%$ were illiterate. Half of the patients (49.8\%) were included in the educational program with no referring personnel while $34.7 \%$ were referred by a doctor, $14.6 \%$ were referred by a health educator, and $0.9 \%$ were referred by a nurse. Furthermore, there was a statically significant difference in patients of PDEP when compared to non-PDEP, in terms of educational status $(\mathrm{P}$-value $=0.036)$, presence of a caregiver $(\mathrm{P}$-value $=$ 


\section{Cureus}

0.03), and referral personnel (P-value < 0.001) (Table 1). The causes of ESRD were variable; some patients had one single cause while some had a combination of causes (Figure 1). A combination of diabetes mellitus (DM) and hypertension (HTN) was the most common cause of ESRD (68.5\%). This was followed by HTN (14.6\%), other conditions (10.8\%), and DM (2.8\%).

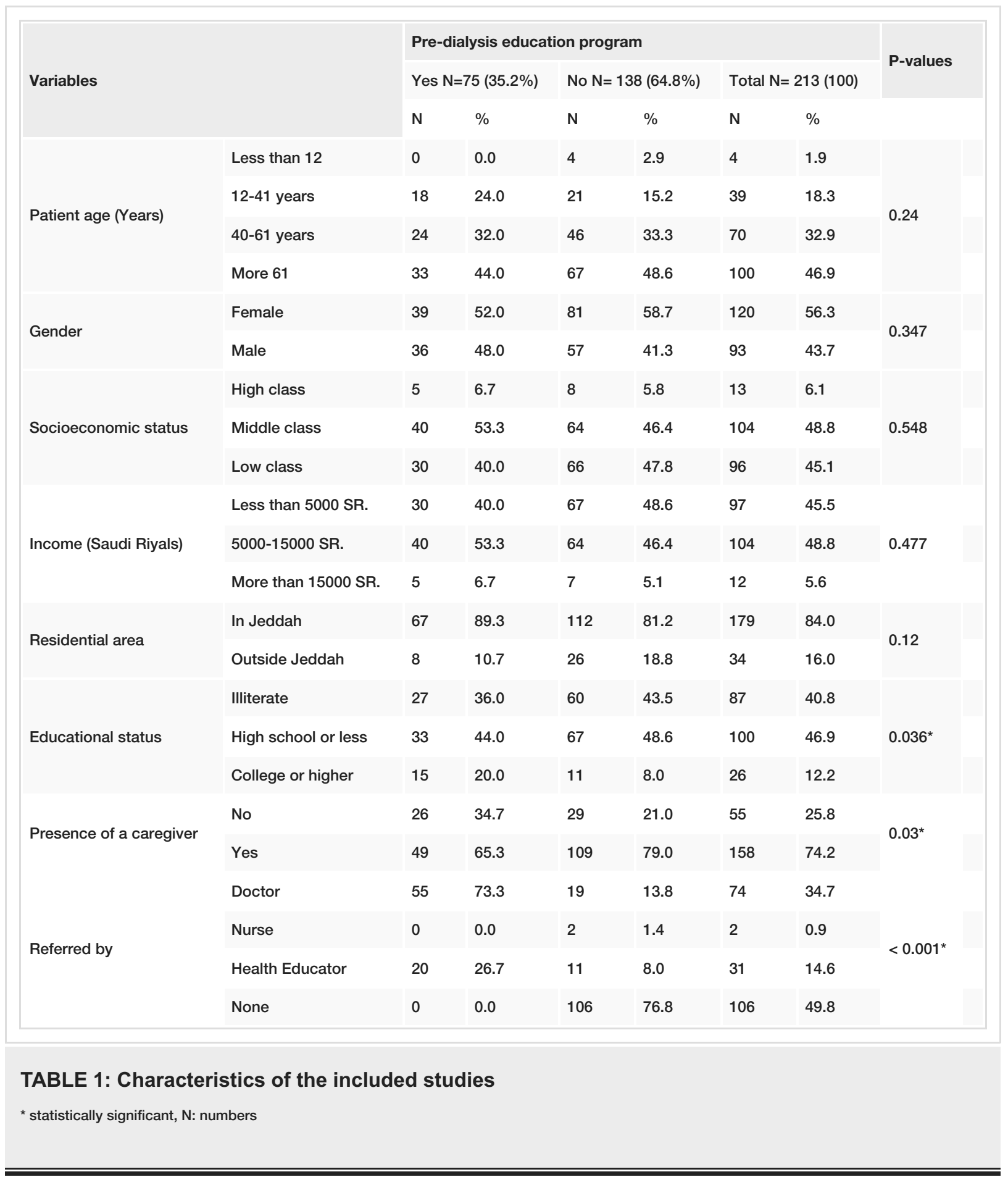




\section{Cureus}

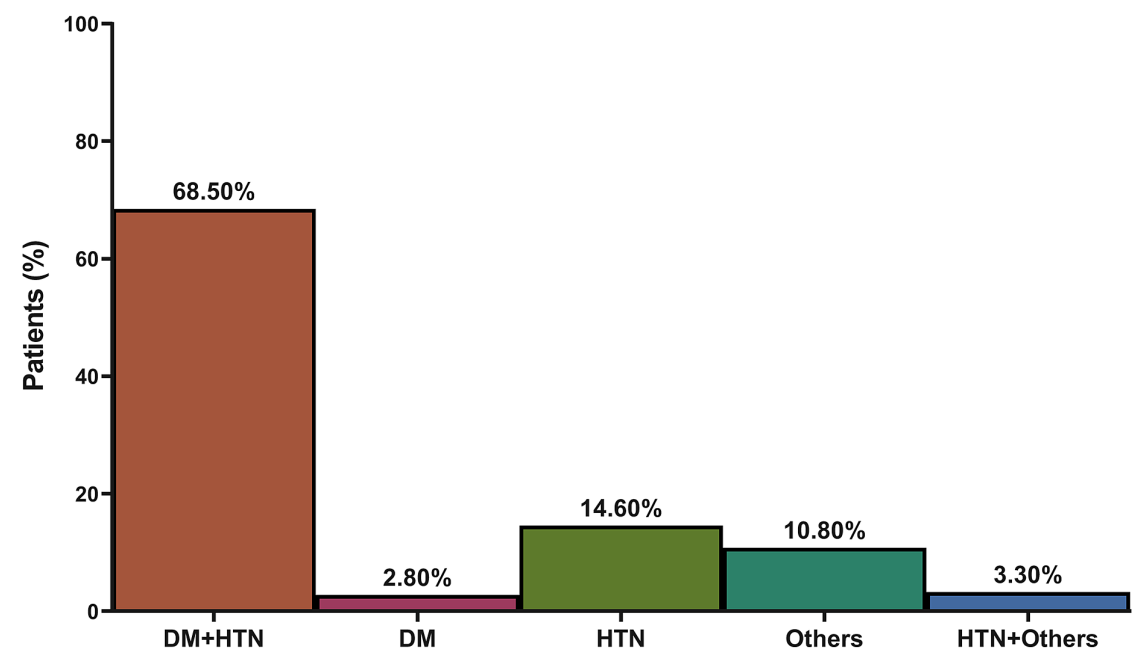

FIGURE 1: Distribution of end-stage renal disease causes among the included patients

DM: diabetes mellitus; HTN: hypertension

\section{Pre-dialysis education and renal replacement therapy mode}

The PDEP was introduced to 75 patients at the beginning of the program; out of those, $57.3 \%$ decided to perform hemodialysis and $42.7 \%$ decided to go with the peritoneal dialysis option. Overall, HD was performed in 168 patients as compared to 45 ones with PD. The introduction of the PDEP was associated with a significant reduction in HD rates [OR $(95 \% \mathrm{CI})=0.11(0.05-0.24)$; P-value < 0.001$]$ (Table 2$)$.

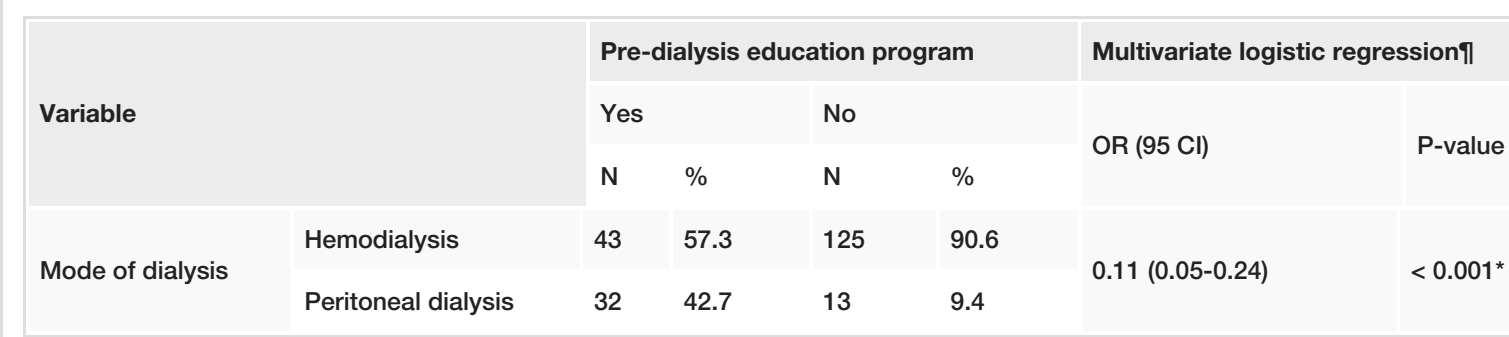

\section{TABLE 2: The impact of pre-dialysis education on the mode of renal replacement therapy}

* Statistically significant; ๆ Effect of Pre-dialysis education on the odds of choosing peritoneal dialysis, where the data were adjusted for patients age, gender, socioeconomic status, and education.

$\mathrm{N}$ : numbers, OR: odds ratio

Hemodialysis patients showed longer mean admission days (3.7 \pm 3.8$)$ as compared to PD patients $(2.4 \pm 1.6)$. Similarly, the frequency of emergency visits was higher in HD patients $(16.9 \pm 14.2)$ as compared to their peers with PD (14.4 \pm 12.6$)$. Moreover, there was a significant increase in admission days for patients undergoing $\mathrm{HD}$ as compared to those having PD [OR $(95 \% \mathrm{CI})=1.27(1.07-1.51)$; P-value $=0.007]$ (Table 3). On one hand, infection rates were higher among the HD group, where $57.70 \%$ of the patients had a central line-associated bloodstream infection (CLABSI). On the other hand, $81.80 \%$ of the PD group did not have any type of infections while $15.90 \%$ had other infections and $2.30 \%$ had peritonitis (Figure 2). 


\section{Cureus}

\begin{tabular}{|c|c|c|c|c|c|c|}
\hline \multirow{3}{*}{ Variables } & \multicolumn{4}{|c|}{ Mode of dialysis } & \multicolumn{2}{|c|}{ Multivariate logistic regressionๆ } \\
\hline & \multicolumn{2}{|c|}{ Hemodialysis } & \multicolumn{2}{|c|}{ Peritoneal dialysis } & \multirow{2}{*}{ OR (95 Cl) } & \multirow{2}{*}{ P-value } \\
\hline & Mean & SD & Mean & SD & & \\
\hline Admission (days) & 3.7 & 3.8 & 2.4 & 1.6 & $1.27(1.07-1.51)$ & $0.007^{*}$ \\
\hline Emergency visits & 16.9 & 14.2 & 14.4 & 12.6 & $1.01(0.99-1.04)$ & 0.397 \\
\hline
\end{tabular}

\section{TABLE 3: Impact of dialysis time on patients' outcomes}

$\mathrm{Cl}$ : confidence interval, OR: odds ratio, SD: standard deviation; * statistically significant; ๆ Effect of dialysis type (hemodialysis) on different patients' outcomes, where the data were adjusted for patients age, gender, socioeconomic status, and education

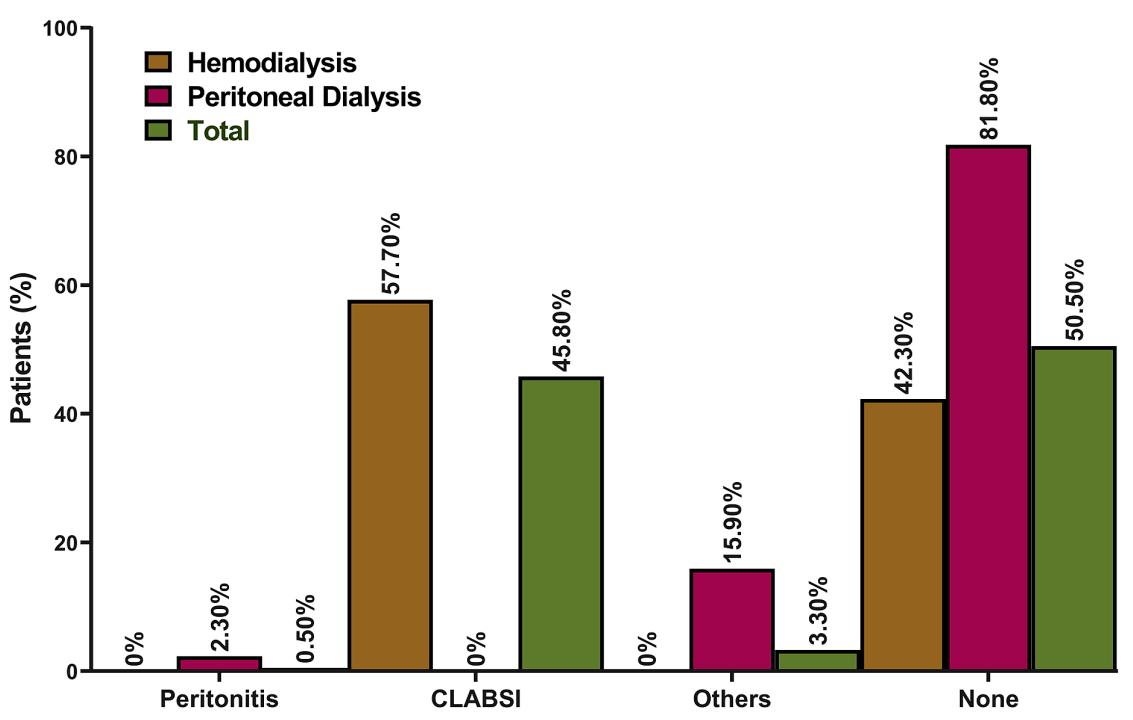

\section{FIGURE 2: Distribution of infections encountered (according to the} dialysis mode)

CLABSI: central line-associated bloodstream infection

\section{Discussion}

PDEP elucidates the overall picture of PD and simplifies the better outcomes that could be obtained from conducting PD over HD. In our study, 75 ESRD patients received a PDEP. Of these patients, $42.7 \%$ of patients preferred PD, with an estimated negative statistical significance of PDEP on conducting HD. Kutner et al. reported that only $10.9 \%$ of the patients responded to the PDEP program, which is lower than our reported rate [23]. The authors justified this low rate by the shortage of content and duration information regarding $\mathrm{PD}$ or that the patients were less satisfied with the process. The authors of the same study also did not find any significance in discussing PD with patients 12 months before initiating regular treatment on preferring PD over HD. On the other hand, almost all previous studies have reported that pre-dialysis education impacted the overall rates of preferring PD and self-care. Ribitsch et al. conducted a case-control study and used a PDEP program as the intervention for the case group while the control did not receive any education [24]. The authors reported that $54.3 \%$ of the patients in the case group chose to initiate their RRT with PD while only $28 \%$ of patients in the control group preferred the same modality. The authors, however, excluded patients with emergencies and those referred to the center at a late stage. The importance of performing PD and self-care has been indicated with similar studies that have also reported similar rates. In a big study conducted by the National Pre-ESRD Education Initiative, which enrolled 15,000 ESRD patients and provided them with PDEP, the results showed that $45 \%$ of these patients preferred PD while the rest preferred HD [25]. The same rate was also reported by Little et al., which investigated the results of 254 patients [26]. Additionally, Manns et al. conducted a randomized trial and found that $82.1 \%$ in the intervention group chose PD, which was significant than the standard care group [27]. 
that the PDEP and non-PDEP patients' age was statistically non-significant. However, Ribitsch et al. reported that initiating RRT was much observed among younger patients with fewer comorbidities and more social activities while older patients preferred HD due to more morbidities and more spare time to regularly visit the dialysis centers [24]. Therefore, every group of patients would choose the most suitable modality that meets their needs and makes them comfortable [28]. Additionally, the early initiation of the PDEP program, while CKD patients are still stable, has a positive outcome on the preference of self-care modalities over the center-based ones together with other outcomes as the frequency of hospitalization and the deterioration level of the case with regards to ESRD [26]. Moreover, using PD as the only self-care modality might be a limitation for many patients, and therefore, other self-care approaches should be provided and discussed in the PDEP programs [29]. Another reason is that such educational programs provide equal insights about the two modalities and some patients are more satisfied with their current regimens than going through changes and future experiments [29]. Finally, we also noticed that CLABSI was a significant factor in preferring HD over PD compared to other causes, which were associated with preferring PD over HD.

Our analysis results showed that PDEP was significantly associated with lower HD rates. Moreover, favorable outcomes, including reduced mean admission days and emergency visits in patients contributed to preferring PD over HD. Many studies have also reported that PD has favorable outcomes, as the fewer overall costs improve the QoL, and the increased patients' satisfaction with PD despite showing that PD and HD were similar in terms of infections and complications [30]. Therefore, we recommend that PDEP programs should be simpler by delivering more persuasive information about the advantages of self-care and PD over center-based HD.

Missing data from some patients' profiles might have been the only limitation in our study. However, whenever data was missing, patients were contacted by telephone or by recalling for a direct interview, and most of the missing information was filled.

\section{Conclusions}

PDEP might have a positive impact on patients preferring PD over HD. Moreover, patients with infections and increased hospital and emergency admission periods were more likely to prefer HD over PD. The value of educational programs among dialysis patients is underestimated and has a very strong impact on their lives when done in a constructive and informative way.

\section{Additional Information \\ Disclosures}

Human subjects: Consent was obtained by all participants in this study. King Fahad Armed Forces Hospital issued approval NA. Animal subjects: All authors have confirmed that this study did not involve animal subjects or tissue. Conflicts of interest: In compliance with the ICMJE uniform disclosure form, all authors declare the following: Payment/services info: All authors have declared that no financial support was received from any organization for the submitted work. Financial relationships: All authors have declared that they have no financial relationships at present or within the previous three years with any organizations that might have an interest in the submitted work. Other relationships: All authors have declared that there are no other relationships or activities that could appear to have influenced the submitted work.

\section{Acknowledgements}

We would like to thank Dr. Suleiman Al Rezgan, Director of the Nephrology Department, for his continuous support and supervision of this work and of the peritoneal dialysis clinic. We would also like to give our thanks to Mr. Ahmad Asmar, Baxter Company, for his support. Lastly, we give our appreciation to Krishann Baguio, the nurse responsible for caring for patients in the Peritoneal Dialysis Clinic and Department; without her continuous support to patients, our education system would be affected severely.

\section{References}

1. Levin A: The advantage of a uniform terminology and staging system for chronic kidney disease (CKD) . Nephrol Dial Transplant. 2003, 18:1446-1451. 10.1093/ndt/gfg241

2. Hemodialysis in the KSA. (2017). Accessed: 2020: https://saudidonation.org/en/annual-reports/.

3. Peritoneal dialysis in KSA. (2017). Accessed: September 13, 2020: https://saudidonation.org/en/annualreports/.

4. Wauters J-P, Uehlinger D: Non-medical factors influencing peritoneal dialysis utilization: the Swiss experience. Nephrol Dial Transplant. 2004, 19:1363-1367. 10.1093/ndt/gfh090

5. Collins AJ, Hao W, Xia H, Ebben JP, Everson SE, Constantini EG, Ma JZ: Mortality risks of peritoneal dialysis and hemodialysis. Am J Kidney Dis. 1999, 34:1065-1074. 10.1016/s0272-6386(99)70012-0

6. Merkus MP, Jager KJ, Dekker FW, et al.: Quality of life over time in dialysis: the Netherlands Cooperative Study on the Adequacy of Dialysis. Kidney Int. 1999, 56:720-728. 10.1046/j.1523-1755.1999.00563.x

7. Lee H, Manns B, Taub K, Ghali WA, Dean S, Johnson D, Donaldson C: Cost analysis of ongoing care of patients with end-stage renal disease: the impact of dialysis modality and dialysis access. Am J Kidney Dis. 
2002, 40:611-622. 10.1053/ajkd.2002.34924

8. Lameire N, Van Biesen W: The pattern of referral of patients with end-stage renal disease to the nephrologist--a European survey. Nephrol Dial Transplant. 1999, 14:16-23. 10.1093/ndt/14.suppl 6.16

9. Winkelmayer WC, Glynn RJ, Levin R, Owen W Jr, Avorn J: Late referral and modality choice in end-stage renal disease. Kidney Int. 2001, 60:1547-1554. 10.1046/j.1523-1755.2001.00958.x

10. Diaz-Buxo JA: Early referral and selection of peritoneal dialysis as a treatment modality . Nephrol Dial Transplant. 2000, 15:147-149. 10.1093/ndt/15.2.147

11. Gómez CG, Valido P, Celadilla O, et al.: Validity of a standard information protocol provided to end-stage renal disease patients and its effect on treatment selection. Perit Dial Int. 1999, 19:471-477. 10.1177/089686089901900511

12. Heaf J: Underutilization of peritoneal dialysis. JAMA. 2004, 291:740-742. 10.1001/jama.291.6.740

13. Van Biesen W, Vanholder RC, Veys N, Dhondt A, Lameire NH: An evaluation of an integrative care approach for end-stage renal disease patients. J Am Soc Nephrol. 2000, 11:116-125.

14. Mendelssohn DC, Pierratos A: Reformulating the integrated care concept for the new millennium. Perit Dial Int. 2002, 22:5-8. 10.1177/089686080202200101

15. Golper TA: Learning about the practice of peritoneal dialysis. Kidney Int. 2009, 76:12-14. 10.1038/ki.2009.65

16. Mehrotra R, Marsh D, Vonesh E, Peters V, Nissenson A: Patient education and access of ESRD patients to renal replacement therapies beyond in-center hemodialysis. Kidney Int. 2005, 68:378-390. 10.1111/j.15231755.2005.00453.x

17. Spigolon DN, de Moraes TP, Figueiredo AE, et al.: Impact of pre-dialysis care on clinical outcomes in peritoneal dialysis patients. Am J Nephrol. 2016, 43:104-111. 10.1159/000444401

18. Isnard Bagnis C, Crepaldi C, Dean J, et al.: Quality standards for predialysis education: results from a consensus conference. Nephrol Dial Transplant. 2015, 30:1058-1066. 10.1093/ndt/gfu225

19. Team RC: R: A Language and Environment for Statistical Computing . R Foundation for Statistical Computing, Vienna, Austria; 2017.

20. R Commander. (2020). http://cran.ma.imperial.ac.uk/web/packages/Rcmdr/Rcmdr.pdf? utm_campaign=share\&utm_medium=email\&utm_source=email_share....

21. Package IM, glm2. Fitting generalized linear models . (2018). Accessed: 2020: https://cran.rproject.org/web/packages/glm2/glm2.pdf.

22. Jiang H, Kulkarni PM, Mallinckrodt CH, Shurzinske L, Molenberghs G, Lipkovich I: Covariate adjustment for logistic regression analysis of binary clinical trial data. Stat Biopharm Res. 2017, 9:126-134. 10.1080/19466315.2016.1234973

23. Kutner NG, Zhang R, Huang Y, Wasse H: Patient awareness and initiation of peritoneal dialysis . Arch Intern Med. 2011, 171:119-124. 10.1001/archinternmed.2010.361

24. Ribitsch W, Haditsch B, Otto R, et al.: Effects of a pre-dialysis patient education program on the relative frequencies of dialysis modalities. Perit Dial Int. 2013, 33:367-371. 10.3747/pdi.2011.00255

25. Golper T: Patient education: can it maximize the success of therapy? . Nephrol Dial Transplant. 2001, 16:2024. 10.1093/ndt/16.suppl_7.20

26. Little J, Irwin A, Marshall T, et al.: Predicting a patient's choice of dialysis modality: experience in a United Kingdom renal department. Am J Kidney Dis. 2001, 37:981-986. 10.1016/S0272-6386(05)80014-9

27. Manns BJ, Taub K, VanderStraeten C, Jones H, Mills C, Visser M, Mclaughlin K: The impact of education on chronic kidney disease patients' plans to initiate dialysis with self-care dialysis: a randomized trial. Kidney Int. 2005, 68:1777-1783. 10.1111/j.1523-1755.2005.00594.x

28. Wuerth D, Finkelstein S, Schwetz O, Carey H, Kliger AS, Finkelstein FO: Patients' descriptions of specific factors leading to modality selection of chronic peritoneal dialysis or hemodialysis. Perit Dial Int. 2001, 22:184-190. 10.1177/089686080202200204

29. Goovaerts T, Jadoul M, Goffin E: Influence of a pre-dialysis education programme (PDEP) on the mode of renal replacement therapy. Nephrol Dial Transplant. 2005, 20:1842-1847. 10.1093/ndt/gfh905

30. Mehrotra R, Chiu Y-W, Kalantar-Zadeh K, Bargman J, Vonesh E: Similar outcomes with hemodialysis and peritoneal dialysis in patients with end-stage renal disease. Arch Intern Med. 2011, 171:110-118. 10.1001/archinternmed.2010.352 